\title{
CULTIVO DE Spirulina sp. LEB 18 EM FOTOBIORREATOR TUBULAR HORIZONTAL PARA BIOFIXAÇÃO DE $\mathrm{CO}_{2}$
}

\author{
A. P. A. CASSURIAGA ${ }^{1}$, E. G. MORAIS ${ }^{1}$, B.C. FREITAS $^{1}$ e J.A.V. COSTA $^{1}$ \\ ${ }^{1}$ Universidade Federal do Rio Grande, Escola de Química e Alimentos \\ E-mail para contato: jorgealbertovc@terra.com.br
}

\begin{abstract}
RESUMO - O potencial biotecnológico das microalgas vem sendo estudado devido às diversas substâncias sintetizadas por estes micro-organismos como proteínas, carboidratos e lipídios. O objetivo do trabalho foi determinar parâmetros cinéticos de crescimento e caracterizar a biomassa de Spirulina sp. LEB 18 cultivada utilizando $\mathrm{CO}_{2}$ como fonte de carbono. O cultivo foi realizado em fotobiorreator tubular horizontal equipado com air-lift sob condições controladas de luz e temperatura, até a fase estacionária de crescimento. Foi utilizado meio Zarrouk com fonte de $\mathrm{N}_{2}$ reduzida em $50 \%$ e fonte de $\mathrm{C}$ substituída por $10 \%$ de $\mathrm{CO}_{2}$. A concentração celular máxima obtida foi 1,06 g.L. $\mathrm{L}^{-1}$, produtividade 0,02 g.L.d $\mathrm{d}^{-1}$ e biofixação diária máxima $160 \mathrm{mg} \cdot \mathrm{L} \cdot \mathrm{d}^{-1}$. A biomassa apresentou teor de proteinas, carboidratos, cinzas e lipidios, 47,3\%, 13,4\%, 12,3\% e 11,7\%, respectivamente, demonstrando que Spirulina sp. LEB 18 possui potencial para desenvolvimento de um processo que resulta na mitigação de $\mathrm{CO}_{2} \mathrm{e}$ uma variedade de biocompostos.
\end{abstract}

\section{INTRODUÇÃO}

As aplicações ambientais das microalgas incluem a biofixação de $\mathrm{CO}_{2}$, remoção de matéria orgânica e metais tóxicos de efluentes, produção de biocombustíveis como biodiesel e bioetanol (LACERDA \& CACIA, 2013). Muitas espécies podem ser induzidas a acumular diversos compostos (SOUSA et al; 2014), como carboidratos, proteínas e lipídios (MORIOKA et al., 2014; HULTBERG et al, 2014). Estudos envolvendo o uso de microalgas vêm se intensificando devido ao potencial que apresentam, sendo uma fonte renovável que não compete diretamente com a produção de alimentos e por possibilitar a obtenção combustíveis com menor impacto ambiental quando comparado aos combustíveis fósseis (SANTOS, 2013).

A biofixação de $\mathrm{CO}_{2}$ por microalgas é uma tecnologia promissora para a redução das concentrações dos gases de efeito estufa (GEE), principalmente $\mathrm{CO}_{2}$. Neste contexto, pesquisas estão sendo desenvolvidas a fim de identificar espécies de microalgas tolerantes a altas concentrações destes gases, e para definição de parâmetros de processo que maximizem as taxas de crescimento e biofixação de $\mathrm{CO}_{2}$ (SINGH \& AHLUWALIA, 2012). Vários métodos químicos, físicos e biológicos têm sido aplicados para redução do $\mathrm{CO}_{2}$ e o método biológico com microalgas é considerado uma das abordagens mais eficazes para a fixação deste gás (DEMIRBAS, 2011). O cultivo de microalgas, comparado ao de vegetais superiores apresenta vantagens como possuir baixos custos para a colheita e transporte, podem ser cultivados em locais inóspitos, não dependem de grandes quantidades de água para cultivo e apresentam resultados imediatos de biofixação (HENRIKSON, 1994). 


\section{9 a 22 de outubro de 2014 \\ Florianópolis/SC}

O nutriente que está em maior quantidade no cultivo de microalgas é o carbono, pois as células apresentam em sua composição cerca de $50 \%(\mathrm{w} / \mathrm{w})$ deste elemento, sendo que este pode se fornecido como $\mathrm{CO}_{2}$, carbonato ou bicarbonato (BORGES et al., 2013). Nos cultivos para mitigação de $\mathrm{CO}_{2}$ a fonte de carbono do meio pode ser substituída pelo $\mathrm{CO}_{2}$ gerado a partir da queima dos combustíveis fósseis, que pode ser captado direto da fonte emissora (BENEMANN, 1993). Esta é uma vantagem adicional a aplicação destes micro-organismos para este fim, visto que além da redução dos GEE ainda há a redução nos custos de produção de biomassa (MORAIS; COSTA, 2007).

O crescimento microalgal é resultado da interação entre fatores biológicos, físicos e químicos. A composição bioquímica da biomassa das microalgas é determinada pela espécie e fatores ambientais como a intensidade de luz, temperatura, $\mathrm{pH}$ e composição do meio de cultivo (FERREIRA et al, 2013). A manipulação de condições de cultivo como presença ou ausência de determinados nutrientes, pode estimular a biossíntese de compostos específicos que estes micro-organismos reagem a variações do meio exterior com alterações intracelulares (NELSON; COX, 2011). A Spirulina é conhecida devido aos diversos compostos que podem ser obtidos através da sua biomassa. Esta microalga vem sendo muito investigada devido à capacidade de se desenvolver em condições ambientais adversas, característica desejável para o aumento de escala. A procura por segurança energética tem feito com que a maioria dos países empenhe-se na busca por fontes alternativas de energia, a fim de reduzir problemas econômicos, sociais e ambientais. Espera-se que a biomassa microalgal, venha a tornar-se um dos principais recursos renováveis na produção de alimentos, materiais, produtos químicos, combustíveis e energia (BORGES et al., 2010).

Neste contexto, o trabalho teve como objetivo determinar parâmetros cinéticos de crescimento e caracterizar a biomassa de Spirulina sp. LEB 18 cultivada utilizando $\mathrm{CO}_{2}$ como fonte de carbono.

\section{MATERIAL E MÉTODOS}

\subsection{Micro-organismo e Condições de Cultivo}

A microalga Spirulina sp. LEB 18 (MORAIS et al., 2008), foi cultivada em meio Zarrouk, contendo $\left(\mathrm{g} \mathrm{L}^{-1}\right): \mathrm{NaHCO}_{3}, 16,8 ; \mathrm{NaNO}_{3}, 2,5 ; \mathrm{K}_{2} \mathrm{HPO}_{4}, 0,5 ; \mathrm{K}_{2} \mathrm{SO}_{4}, 1,0 ; \mathrm{NaCl}, 1,0 ; \mathrm{Mg} . \mathrm{SO}_{4} .7 \mathrm{H}_{2} \mathrm{O}$, 0,$2 ; \mathrm{CaCl}_{2}, 0,04 ; \mathrm{FeSO}_{4} .7 \mathrm{H}_{2} \mathrm{O}, 0,01 ;$ EDTA, 0,08 e micronutrientes. Neste experimento, a fonte de nitrogênio foi reduzida em $50 \%$ e a fonte de carbono foi substituída por $\mathrm{CO}_{2}$ comercial a $10 \%(\mathrm{v} / \mathrm{v})$ em uma mistura com ar a $90 \mathrm{~mL}_{\text {mistura }} / \mathrm{min}$. A injeção ocorreu a cada $40 \mathrm{~min}$ na fase clara durante 5 min. Os cultivos foram realizados em fotobiorreator tubular horizontal (Figura 1) equipado com airlift de 150L, volume útil de 130L, com concentração inicial de cultivo de 0,2 g.L $\mathrm{L}^{-1}$. Os experimentos foram conduzidos em estufa termostatizada a $30^{\circ} \mathrm{C}$ com controle de iluminância $\left(43,2 \mu \mathrm{mol} . \mathrm{m}^{-2} . \mathrm{s}^{-1}\right)$, fotoperíodo $12 \mathrm{~h}$ claro/escuro e mantidos até a fase estacionária de crescimento. 


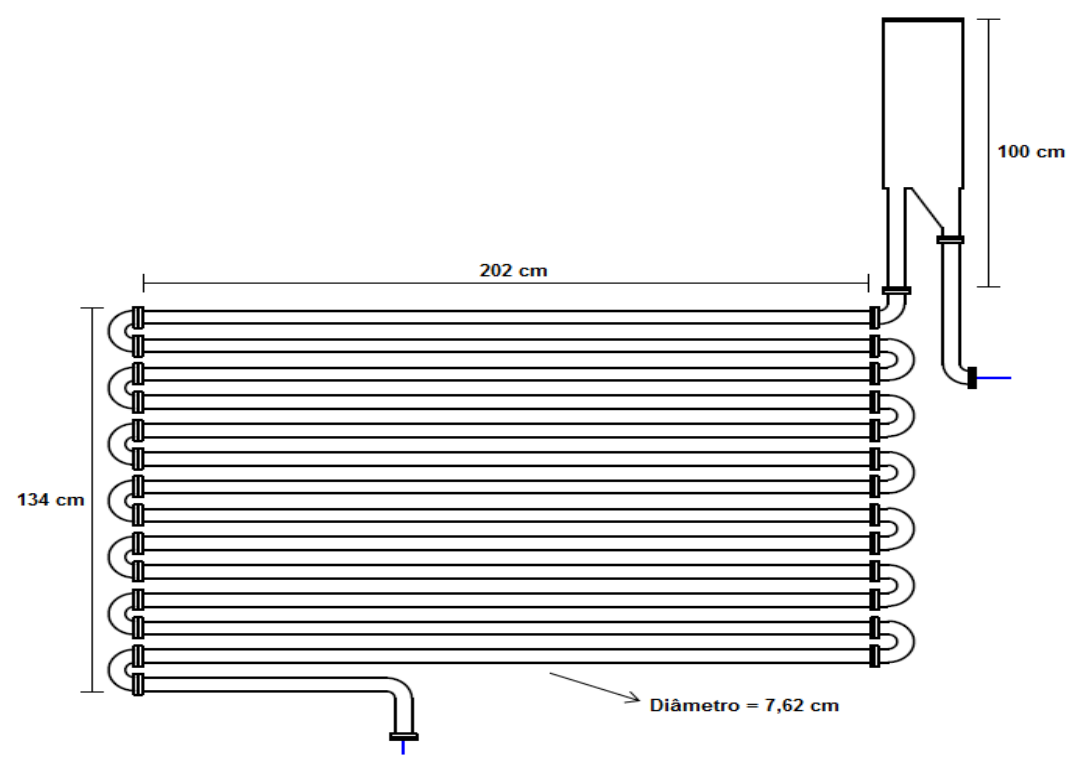

Figura 1 - Esquema biorreator tubular horizontal equipado com air-lift.

\subsection{Caracterização da Biomassa}

A biomassa obtida foi centrifugada a 15200x $g$ por 15 min e liofilizada, logo após reidratada e submetida a sonda ultrassônica por 10 min em pulsos de 10s, obtendo o extrato microalgal. A partir deste extrato foram realizadas análises de carboidratos segundo Dubois et al. (1956) e teor de proteínas pelo método proposto por Lowry et al. (1951) utilizando albumina como padrão. Os lipídios foram quantificados a partir da biomassa liofilizada utilizando método de Folch et al. (1957). O teor de umidade e cinzas de acordo com AOAC (2000).

A fim de realizar comparação de resultados foi utilizada uma biomassa de Spirulina sp. LEB 18 produzida comercialmente pela empresa Olson microalgas. Esta biomassa foi cultivada em fotobiorreatores raceway sob condições outdoor em estufa de filme transparente. Nesta biomassa as mesmas quantificações foram realizadas.

\subsection{Delineamento experimental}

Diariamente foi monitorado o crescimento das microalgas através da medida da concentração celular, determinada pela densidade óptica das culturas em espectrofotômetro a $670 \mathrm{~nm}$. A concentração celular foi obtida através de curva de crescimento relacionando massa seca e densidade óptica (COSTA et al., 2002). Também a cada 24 h foi realizada a medida do $\mathrm{pH}$ dos cultivos em pHmetro digital.

Neste ensaio foram determinados os parâmetros concentração celular máxima ( $\mathrm{X}_{\text {máx }}$ ), produtividade máxima $\left(\mathrm{P}_{\text {máx }}\right)$, velocidade específica máxima de crescimento $\left(\mu_{\text {máx }}\right)$, e tempo de geração (tg). A produtividade $\left(P\right.$, g.L. $\left.d^{-1}\right)$ foi calculada segundo a equação $P=\left(X-X_{0}\right) /\left(t-t_{0}\right)$. A 
velocidade específica de crescimento $\left(\mu_{\text {máx }}\right)$ foi obtida por regressão exponencial da fase logarítmica de multiplicação celular e o tempo de geração ( $\operatorname{tg}$ ) foi obtido pela razão entre $\ln 2 / \mu_{\text {máx }}$ (MORAIS; COSTA, 2007).

\section{RESULTADOS E DISCUSSÃO}

No experimento com Spirulina sp. LEB 18 utilizando meio Zarrouk com redução na fonte de nitrogênio e substituição da fonte de carbono por $\mathrm{CO}_{2}$, houve uma fase de adaptação de aproximadamente $7 \mathrm{~d}$, conforme Figura 2. Após este período a velocidade de crescimento celular aumentou gradualmente, até o ponto onde manteve-se constante, na etapa de crescimento exponencial entre 12 d e 40 d, até atingir a concentração celular máxima. Neste ponto houve uma desaceleração até o início na fase estacionária, identificada pelo ponto onde a concentração celular se estabilizou. A entrada na fase estacionária para os cultivos microalgais geralmente ocorre devido ao esgotamento de nutrientes ou sombreamento celular (COELHO, 2013). Ao longo dos experimentos, não houve grande variação do pH devido as injeções de $\mathrm{CO}_{2}$, se mantendo entre 7,75 e 8,83.

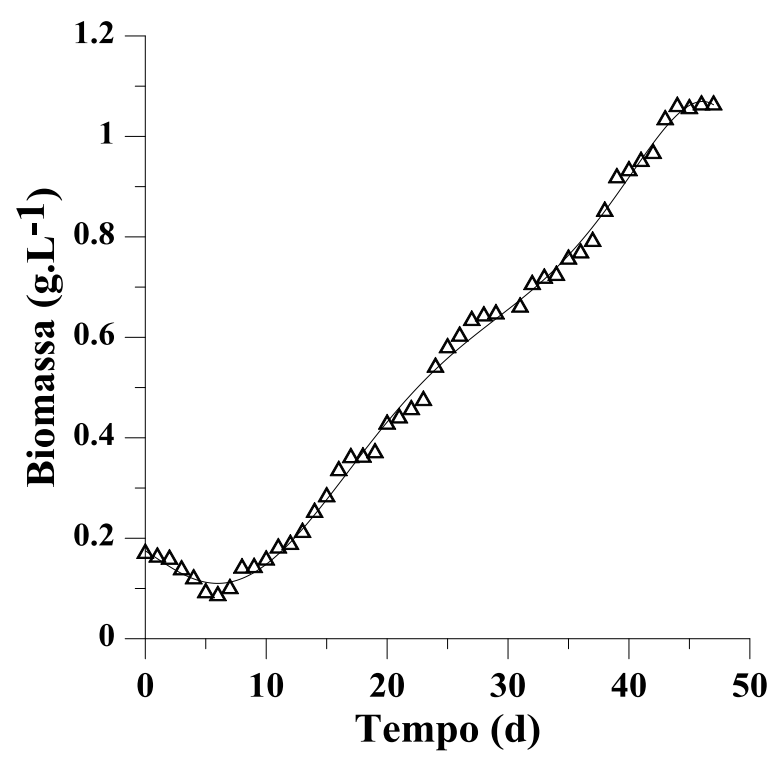

Figura 2 Curva de crescimento da microalga Spirulina sp. LEB $1850 \% \mathrm{~N}, 10 \% \mathrm{CO}_{2}$, meio Zarrouk modificado.

No experimento com a microalga Spirulina sp. LEB 18 os parâmetros cinéticos obtidos ao final dos $47 \mathrm{~d}$ de experimento foram esperados devido às alterações no meio de cultivo conforme Tabela 1. Radmann et al. (2011) ao cultivarem Spirulina sp. LEB 18 em meio Zarrouk utilizando $10 \%$ de $\mathrm{CO}_{2}$ como fonte de carbono, durante $15 \mathrm{~d}$ obtiveram concentração celular máxima de $1,53 \mathrm{~g} . \mathrm{L}^{-1} \mathrm{e}$ velocidade específica máxima de crescimento de $0,22 \mathrm{~d}^{-1}$ resultados superiores aos obtidos no presente estudo. A redução da fonte nitrogênio pode ter influenciado negativamente o desenvolvimento deste micro-organismo, reduzindo a produtividade $\mathrm{e}$, consequentemente, concentração celular máxima ao final do experimento. O nitrogênio é um elemento utilizado pelas células principalmente para formação de proteínas e ácidos nucléicos, sendo essencial ao 
desenvolvimento celular (BEZERRA et al., 2012).

Tabela 1 Parâmetros cinéticos para o cultivo de microalga Spirulina sp. LEB 18 50\% N, 10\% $\mathrm{CO}_{2}$ e biofixação

\begin{tabular}{cc}
\hline Parâmetros Cinéticos & Respostas \\
\hline$X_{\text {máx }}$ & $1,06 \mathrm{~g} \cdot \mathrm{L}^{-1}$ \\
$\mathrm{Tg}$ & $5,39 \mathrm{~d}$ \\
$\mathrm{P}_{\text {máx }}$ & $0,02 \mathrm{~g} . \mathrm{L} \cdot \mathrm{d}^{-1}$ \\
$\mu_{\text {máx }}$ & $0,13 \mathrm{~d}^{-1}$ \\
Biofixação & $160 \mathrm{mg} \cdot \mathrm{L} \cdot \mathrm{d}^{-1}$ \\
\hline
\end{tabular}

A redução na fonte de nitrogênio é uma forma efetiva de melhorar o acúmulo de lipídeos e/ou carboidratos na biomassa microalgal, no entanto o aumento destes componentes na biomassa nem sempre é proporcional ao da produtividade celular. Nas microalgas, a resposta das células às condições ambientais definem os fatores como limitante, quando ocorre a redução da taxa de crescimento ou alguma reação bioquímica sem a necessidade de aclimatação celular, ou estressante, que implica em um desequilíbrio metabólico o qual demanda ajustes bioquímicos antes que as células possam estabelecer um novo estado de crescimento ou biossíntese (RICHMOND, 2008).

O cultivo realizado em biorreator tubular horizontal apresentou taxa de biofixação de $\mathrm{CO}_{2}$ de 160 mg.L.d ${ }^{-1}$, valor inferior ao obtido por Morais e Costa (2007), quando cultivaram a microalga Spirulina sp. LEB 18 em biorreator tubular vertical com injeção de $6 \%$ de $\mathrm{CO}_{2}\left(394 \mathrm{mg} \mathrm{L}^{-1} \mathrm{~d}^{-1}\right)$. O mecanismo de biofixação de $\mathrm{CO}_{2}$ por microalgas é baseado na capacidade que esses microorganismos possuem de realizar fotossíntese. Mudanças nas fontes nutricionais geralmente não afetam a taxa fotossintética de microalgas, no entanto, a alteração na concentração de nutrientes, como $\mathrm{N}$ e P, podem causar a redução na absorção de $\mathrm{CO}_{2}$ (OHSE et al., 2007). Outros fatores como, iluminância, taxa de injeção de $\mathrm{CO}_{2}$ nos cultivos, $\mathrm{pH}$, densidade celular e temperatura também podem determinar um aumento ou redução na biofixação de $\mathrm{CO}_{2}$ (JARENKOW, 2011).

O teor de lipídios encontrado para a biomassa de Spirulina sp. LEB 18 cultivada em biorreator tubular horizontal é cerca de duas vezes maior que os valores encontrados em cultivos comerciais como apresentado na Tabela 2. O tempo de cultivo, até a fase estacionária, e a redução do componente nitrogenado podem ter contribuído para o aumento da concentração lipídica. Na fase de crescimento exponencial geralmente o teor proteico é mais elevado devido à multiplicação celular e a formação dos ácidos nucleicos. A maior produção de lipídios e carboidratos, no entanto, ocorre na fase estacionária como forma de reserva de energia (DUNSTAN, 1993). Já a redução da fonte de nitrogênio em cultivos microalgais é capaz de realizar um desvio no metabolismo das células para a 
produção de lipídios ou carboidratos (LOURENÇO, 2006). No caso do cultivo em biorreator tubular da cepa Spirulina sp. LEB 18 este desvio foi favorável a produção de lipídios, visto que comparandose as concentrações de carboidrato da Tabela 2 pode-se observar que este foi inferior ao obtido no cultivo comercial.

Tabela 2 Teor de carboidratos, lipídios, proteínas e cinzas na biomassa de Spirulina sp. LEB 18 em biorreator tubular e raceway

\begin{tabular}{ccccc}
\hline Amostra & Carboidratos (\%) & Lipídios (\%) & Proteínas (\%) & Cinzas (\%) \\
\hline Tubular & $13,40 \pm 0,49$ & $11,69 \pm 0,76$ & $47,26 \pm 1,10$ & $12,3 \pm 0,8$ \\
Raceway: & $21,68 \pm 0,12$ & $5,74 \pm 0,46$ & $44,69 \pm 0,25$ & $15,6 \pm 0,7$
\end{tabular}
microalgas.

*valores obtidos para a biomassa de Spirulina sp LEB 18, cultivada pela empresa Olson

Com relação ao teor de proteínas e cinzas, os cultivos comercial e em biorreator tubular horizontal mantiveram-se próximos. As variações da composição da biomassa das microalgas cultivadas sob diferentes formas, podem ter ocorrido devido às diferentes configurações dos reatores (volume, agitação e exposição a luz solar) e condições ambientais aos quais foram submetidos os cultivos. As variações de temperatura e luminosidade em condições outdoor podem afetar a composição da biomassa microalgal (RICHMOND, 1986).

A microalga Spirulina sp. possui a capacidade de produzir biomassa rica em biocompostos (proteínas, lipídios e carboidratos), que podem ser utilizados na produção de biocombustíveis, suplementos alimentares, produção de fármacos e cosméticos bem como no sequestro de carbono sendo uma alternativa viável para redução da concentração de $\mathrm{CO}_{2}$ atmosférico.

\section{CONCLUSÃO}

O cultivo da microalga Spirulina sp. LEB 18 realizado em fotobiorreator tubular horizontal equipado com air-lift, com redução da fonte de nitrogênio e substituição da fonte de carbono por $10 \%$ de $\mathrm{CO}_{2}$ no meio, obteve concentração celular máxima 1,06 g.L $\mathrm{L}^{-1}$ e produtividade 0,02 g.L.d ${ }^{-1}$ ao final de $47 \mathrm{~d}$ de cultivo. A microalga produziu biocompostos com aplicabilidade em diversas áreas e a produção de lipídios pode ter sido estimulada pela redução do componente nitrogenado. Spirulina $\mathrm{sp}$ LEB-18 biofixou $160 \mathrm{mg}_{\mathrm{CO} 2} \cdot \mathrm{L}^{-1} \cdot \mathrm{d}^{-1}$ mostrando-se como uma alternativa para a mitigação das emissões antropogênicas de $\mathrm{CO}_{2}$.

\section{AGRADECIMENTOS}

Os autores agradecem a Olson Nutrição, Companhia de Geração Térmica de Energia Elétrica (CGTEE), Capes e Fapergs pelo apoio a este trabalho. 


\section{REFERENCIAS}

A.O.A.C.; Official Methods of Analysis, Association of Official Analytical Chemist EUA, 2000. BENEMANN, J. R.; Utilization of carbon dioxide from fossil fuel - burning power plants with biological system. Energy conversion and management, v. 34, p.999-1004, 1993.

BEZERRA, R.P.; MATSUDO, M.C.; SATO, S.; PEREGO, P.; CONVERTI, A.; CARVALHO, J.C.M.; Effects of photobioreactor configuration, nitrogen source and light intensity on the fedbatch cultivation of Arthrospira (Spirulina) platensis. bioenergetic aspects. Biomass and Bioenergy, v. 37, p. 309-317, 2012.

BORGES, F.C.; TRIERWEILER, J.O.; Proposta de um modelo conceitual de biorrefinaria com estrutura descentralizada. Universidade Federal do Rio Grande do Sul. Escola de Engenharia. Programa de Pós-Graduação em Engenharia Quimica, 2010.

BORGES, J. A. et al.; Spirulina sp. LEB 18 culture using effluent from the anaerobic digestion. Braz. J. Chem. Eng., v.30, n.2, p. 277-288, 2013.

COSTA, J. A. V.; COLLA, L. M.; DUARTE FILHO, P.; KABKE, K.; WEBER, A. Modelling of Spirulina platensis growth in fresh water using response surface methodology. World J Microb Biot, v. 18, p. 603-607, 2002.

COELHO, V.C.; Síntese de Biopolímeros pela Microalga Spirulina sp. LEB-18 cultivada em Diferentes Concentrações de Nutrientes. Dissertação de Mestrado, Universidade Federal do Rio Grande, 2013.

DERMIBAS A.; Biodiesel from oilalgae, biofixation of carbon dioxide by microalgae: A solution to pollution problems. Appl Energy, 2011.

DUBOIS, M., GILLES, K., HAMILTON, J., REBERS, P., \& SMITH, F.; Colorimetric method for determination of sugars and related substances. Analytical Chemistry, v.28(3), p.350-356, 1956.

DUNSTAN, G. A.; Changes in lipid compositions and maximization of the polyunsaturated fatty acid contente of three microalgae grown in mass culture. Journal of Applied Phycology, v.5, p.71-83, 1993.

FERREIRA, S.P.; SOUZA-SOARES, L.; COSTA, J.A.V.; Revisão: microalgas: uma fonte alternativa na obtenção de ácidos gordos essenciais. Rev. de Ciências Agrárias, v.36, n.3, p. 275$287,2013$.

FOLCH, J.; LEES, M.; STANLEY, G. H. S.; J. Biol. Chem. , v.226, n.497, 1957.

HENRIKSON, R.; Microalga Spirulina, superalimento del futuro, Ronore Enterprises. $2^{\mathrm{a}}$ ed. Ediciones Urano, Barcelona, España. p.222, 1994.

HO, S. H.; CHENA, C. Y.; YEHA, K. L.; CHENC, W. M.; LIND, C. Y.; CHANG, J. S. Characterization of photosynthetic carbon dioxide fixation ability of indigenous Scenedesmus obliquus isolates. Biochemical Engineering Journal, v. 53, p. 57-62, 2010.

HULTBERG, B., BERGLUND, M., ANDERSSON, A. AND FRANK, A. ;Elevated plasma homocysteine in alcoholics. Alcoholism: Clinical and Experimental Research, v.17, p.687-689, 2014.

JARENKOW, A.; Influencia da vazão de entrada de ar no crescimento de microalgas em reator tipo air-lift; Trabalho de Diplomação em Engenharia Quimica, Universidade Federal do Rio Grande do Sul, 2011. 
LACERDA, L.M.C.; Otimização de sistemas de microalgas para mitigação de $\mathrm{CO}_{2}$ e produção de biodiesel; Dissertação de Mestrado, Programa de Pós-graduação em engenharia Quimica, Campinas SP, 2013.

LOURENÇO, S.O.; Cultivo de microalgas marinhas: princípios e aplicações. São Carlos: RiMa, p.606, 2006.

MORAIS, M. G.; COSTA, J. A. V.; Biofixation of carbon dioxide by Spirulina sp. and Scenedesmus obliquus cultivated in a three-stage serial tubular photobioreactor. Journal of Biotechnology, v.129, p.439-445, 2007.

MORAIS, M.G.; COSTA, J.A.V.; Bioprocessos para remoção de dióxido de carbono e óxido de nitrogênio por micro-algas visando a utilização de gases gerados durante a combustão do carvão. Quím. Nova. v.31, p.1038-1042, 2008.

MORIOKA, L.R.I.; MATOS, A.P.; OLIVO, G.; SANT'ANNA, E.S.; Floculação de Chlorella sp. produzida em concentrado de dessalinização e estudo de método de extração de lipídeos intracelulares. Quím. Nova, v.37, p.44-49, 2014.

NELSON, D.L.; COX, LEHNINGER, M.M.; Lehninger: Princípios de Bioquímica. $5^{\circ}$ Ed. São Paulo: Sarvier, 2011.

OHSE, S.; DERNER, R.B.; OZERIO, R.A.; CUNHA, P.C.R.; LAMARCA, C.P.; SANTOS, M.E.; MENDES, L.B.B.; Revision on carbon sequestration by microalgae and forests and the production of lipids for the microalgae; Dissertação de mestrado, Pós-Graduação em Ciência dos Alimentos Universidade Federal de Santa Catarina, Santa Catarina, Brasil, 2007.

RADMAN, E. M., CAMERINI, F.V., SANTOS, T. D., COSTA, J. A. V.; Isolation and application of $\mathrm{SO}_{\mathrm{X}}$ and $\mathrm{NO}_{\mathrm{X}}$ resistant microalgae in biofixation of $\mathrm{CO}_{2}$ from thermoelectricity plants. Energy Conversion and Management, v.52, p.3132-3136, 2011.

RICHMOND, A. Handbook of microalgal culture: biotechnology and applied phycology. London: Blackwell Science, v.15, p.312-351. 2004.

SANTOS, M.O.; Rheological behavior of Chlorella sp. e Scenedesmus sp. cultures in different biomass concentrations. Eng. Agríc., v.33, n.5, p.1063-1071, 2013.

SINGH, U. B.; AHLUWALIA, A. S. Microalgae: a promising tool for carbon sequestration. Mitig Adapt Strateg Glob Change, 2012.

SOUSA, L.L.; HORA, D.S.; SALES, E.A.; PERELO, L.W.; Cultivation of Nannochloropsis sp. in brackish groundwater supplemented with municipal wastewater as a nutrient source. Braz. arch. biol. technol. v.57, n.2, p.171-177, 2014.

ZARROUK, C.; Contribution à l'étude d'une cyanophycée. Influence de diveurs facteurs physiques et chimiques sur la croissance et photosynthese de Spirulina maxima Geitler. Ph.D. Thesis, University of Paris, 1966. 